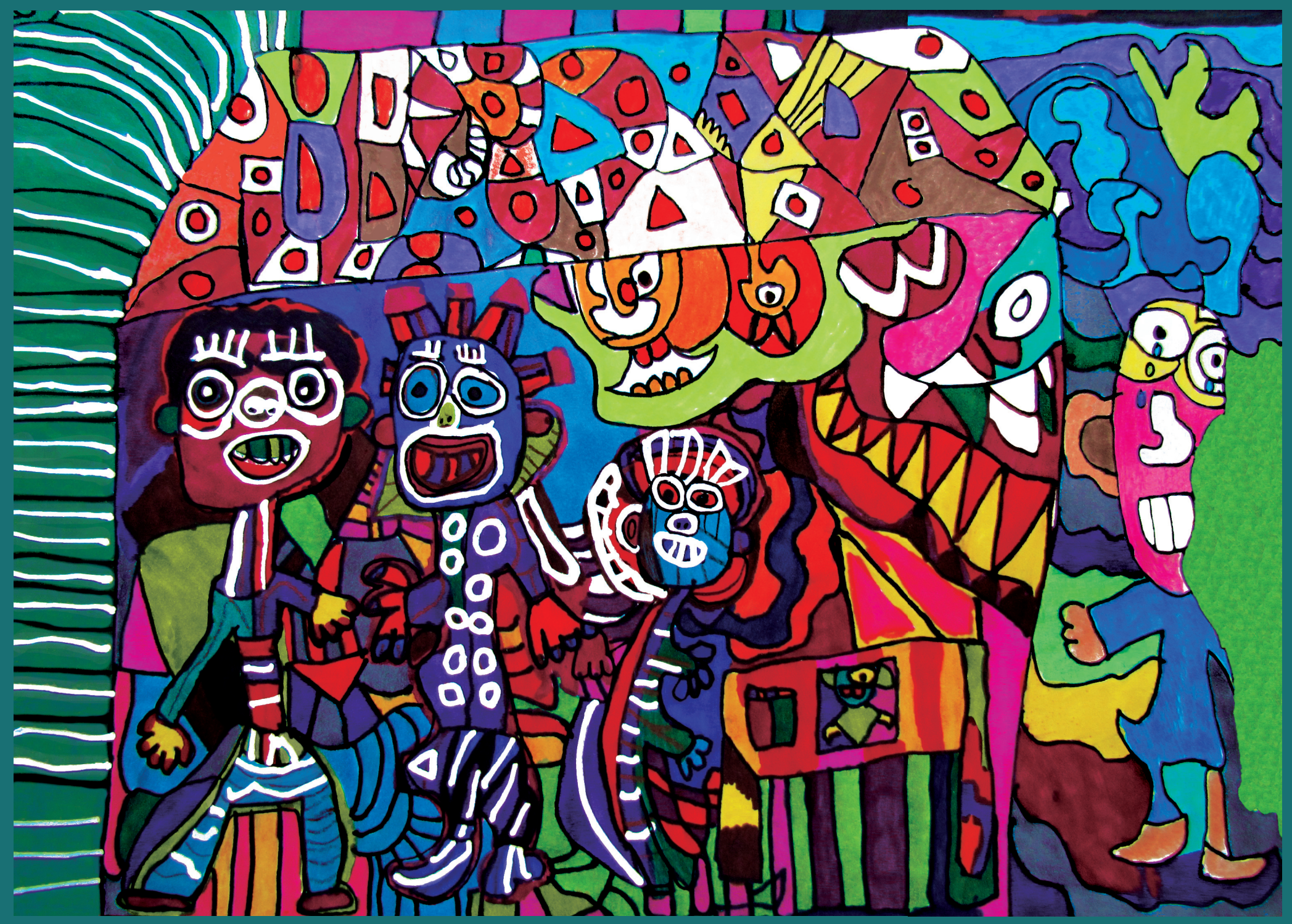

Emilio Morales - Arte sin Fronteras - Programa Talentos Especiales

Martha Lucia Rincón Bustos

Magister en Estructuras y Procesos de Aprendizaje

mlrinconb@unal.edu.co

Doris Lucia Bernal Barrera

Fonoaudiología

dbernalb@unal.edu.co
Diana Sofía Martínez Tintinago

Fonoaudiología

dsmartinezt@unal.edu.co

Lorena Estefanía Pachón Salem

Fonoaudiología

lepachonc@unal.edu.co
Jhezabel Rodero Suarez

Fonoaudiología

jhezabel1409@hotmail.com

Ana Jazmín Uran Loaiza

Fonoaudiología

ajuranl@unal.edu.co 


\section{DIFICULTADES DE APRENDIZAJE ESCOLAR Y TRASTORNO POR DÉFICIT DE ATENCIÓN E HIPERACTIVIDAD (ESTUDIO BIBLIOMÉTRICO)}

\section{School learning disabilities and attention deficit hyperactivity disorder - ADHD - (Bibliographical study)}

Fecha de Recepción: 20 de Abril de 2011 - Fecha de Aprobación: 15 de Septiembre de 2011

RESUMEN

El trastorno por déficit de atención/hiperactividad (TDAH) es una de las alteraciones neurocomportamentales más comunes de la niñez y una de las causas de consulta más frecuentes en la edad escolar, debido al gran impacto que se genera en los procesos del aprendizaje, dadas las implicaciones de las funciones ejecutivas como la memoria y la atención. El presente estudio de tipo bibliográfico tiene como objetivo realizar un análisis acerca del estado actual en que se encuentran las investigaciones con respecto al TDAH y su relación con las dificultades de aprendizaje, con el fin de indagar acerca del rol del fonoaudiólogo en este campo. En general los resultados apuntan hacia una fuerte comorbilidad entre el TDAH y dificultades de aprendizaje además de deficiencias en habilidades cognitivas, procesamiento matemático, lectura, escritura y especialmente el lenguaje.

PALABRAS CLAVE

Déficit de atención, bibliometría

\section{ABSTRACT}

Attention Deficit Hyperactivity Disorder (ADHD) is one of the most common neurobehavioral disorders of childhood and one of the most frequently reported causes of school age because the high impact that is generated in the processes of learning, given the implications of executive functions such as memory and attention. The present bibliographical study aims to perform an analysis about the current state that is research regarding ADHD and its relationship with learning difficulties, in order to inquire about the role of speech-language pathologist in this field. In general, the results point to a strong comorbidity between ADHD, learning disabilities, as well as deficiencies in cognitive abilities, mathematical processing, reading, writing and especially the language.

KEY WORDS

Attention deficit, bibliometrics 


\section{INTRODUCCIÓN}

$\mathrm{E}$ presente artículo con un ánimo, si se quiere ambicioso, muestra los resultados de una cuidadosa búsqueda de artículos de investigación los cuales realizaban un trabajo de campo en población con Trastorno de Déficit de Atención\Hiperactividad (TDAH). El objetivo fue realizar un análisis acerca del estado actual en que se encuentran las investigaciones con respecto al TDAH y su relación con las dificultades de aprendizaje escolar, todo esto con el fin de explorar el campo de acción profesional para los fonoaudiólogos en este panorama.

El interés particular en este trastorno radica en la fuerte asociación que presenta con las dificultades de aprendizaje, entre las cuales podemos destacar la adquisición deficiente de las habilidades necesarias para desarrollar la lectoescritura y otras competencias no solo de la vida escolar, sino de otros contextos $(1,4)$. Partiendo de estas dificultades y su íntima relación con el lenguaje, otro punto de interés es el rol del fonoaudiólogo dentro de los procesos de tratamiento de la población con TDAH, debido a la experticia de este profesional en estas áreas, en las dimensiones comprensivas y expresivas del lenguaje en sus modalidades oral y escrita.

Se iniciará con una breve descripción del TDAH y las dificultades de aprendizaje, y posteriormente se profundizara en su fuerte correlación.

Las dificultades de aprendizaje pueden ser definidas teóricamente desde un sentido amplio o estricto; según el sentido amplio un niño tiene una dificultad de aprendizaje si presenta un déficit para aprender significativamente mayor que los niños de su edad (5); y según el sentido estricto son escolares con dificultades para adquirir conocimientos en diversas áreas escolares como la lectura, la escritura, la aritmética y falencia en aspectos comportamentales debido a una disfunción cerebral mínima o a un disturbio emocional (6). Para esta investigación, se tomará como base la segunda definición expuesta.

El trastorno por déficit de atención/hiperactividad es considerado actualmente como una alteración del neurodesarrollo infantil caracterizada por la inmadurez de los centros neuronales que regulan el funcionamiento ejecutivo (7), asociado a una disfunción cerebral mínima (4) con etiología de base biológica y/o genética, como es el caso del consumo de drogas y alcohol durante el embarazo, presencia de riesgo en la maduración fetal, sobreestimulación prenatal, antecedentes familiares de TDAH y factores psicosociales adversos, entre otros. Algunas teorías explicativas apuntan a la sobrexcitación o inhibición de 3 neurotransmisores específicos: noradrenalina, acetilcolina y dopamina (7); de la misma manera otras hipótesis sugieren disfunción en el lóbulo frontal relacionado con las funciones ejecutivas (8), y fallas en regiones parietales del hemisferio izquierdo, así como sobrexcitación reticular e hiperactividad en el cuerpo estriado; cabe mencionar aquí que aunque los avances investigativos son notables existe mucho campo por explorar en la búsqueda de respuestas etiológicas.

El TDAH se caracteriza principalmente por una enorme dificultad para focalizar el esfuerzo mental y la atención en estímulos significativos durante un periodo de tiempo contiguo y al mismo tiempo excluir otros (9) personalidad dispersa, impulsiva y desorganizada (10) y usualmente es acompañado por un exceso de actividad motora (11); los niños con TDAH pasan poco tiempo en la misma posición y en la misma actividad, presentando variabilidades de acuerdo a la complejidad del cuadro clínico y demostrando un fuerte impacto en las dimensiones sociales y familiares. Estos síntomas deben presentarse antes de los 7 años de edad y manifestarse en más de un lugar (casa, colegio) durante un período superior a 6 meses impactando negativamente en las actividades sociales, académicas y ocupacionales (12).

Usualmente los niños con TDAH presentan un Coeficiente Intelectual (CI) normal, sin embargo es común que se evidencien deficiencias cognitivas, de lenguaje y de cálculo matemático, producto de las alteraciones en las funciones ejecutivas y metacognitivas (13) que no dan respuesta al origen del trastorno sino que más bien son consecuencia de este. Estas manifestaciones, como es de esperar, generan dificultades en el aprendizaje las cuales se hacen evidentes en falencias de habilidades como escuchar, entender, retener información en la memoria, elaborar un discurso, generar respuestas escritas u orales (14).

Los criterios del DSM-IV para el diagnóstico y la categorización del TDAH están de acuerdo a la predominancia de los siguientes signos:

1. Déficit de atención: Fallas para centrar la atención profundamente en los detalles, dificultad para: Sostener la atención en las actividades, escuchar cuando se le habla directamente, seguir instrucciones y conversaciones; facilidad para olvidar y distraerse con estímulos externos.

2. Hiperactividad e impulsividad: Intranquilidad persistente, movimientos de un lado a otro en situaciones en las que debe estar quieto, dificultad para jugar tranquilamente, habla excesiva, repuestas precoces (responde incluso antes de que la pregunta haya terminado de ser formulada), dificultad para respetar los turnos de conversación, e interrumpe y molesta otros niños.

3. Mixta: Presenta conductas propias de las categorías anteriores.

Como fue mencionado anteriormente existe una fuerte comorbilidad entre TDAH y las dificultades del aprendizaje (9), dado que existen diversas fallas en sistemas neuronales, por ejemplo los sistemas atencionales y reticulares los cuales se basan en el funcionamiento de las redes encargadas de la activación y alerta que permiten el control de los estímulos internos y externo; las redes implicadas en la orientación hacia los estímulos del entorno definida por la función visual, auditiva y somestésica, y las redes implicadas en la atención selectiva la cual es la encargada de focalizar y mantener la atención ante estímulos determinados filtrando los estímulos irrelevantes; es necesario tener en cuenta que sostener los niveles de concentración resulta ser una operación bastante compleja, en especial para los niños con TDAH, quienes necesitan un apoyo escolar y extraescolar para lograrlo (9).

Un aspecto destacado por investigadores como Virginia Douglas (8), es la importancia de la atención dentro del proceso de aprendiza- 
je, pues esta tiene una función crucial en el procesamiento de información que a su vez está ligado a dificultades de orden cognitivo que impiden el desarrollo de esquemas y estrategias para la resolución de problemas, debilidades en la motivación y deficiencias de carácter metacognitivo e incapacidad para planificar y controlar la acción propia. De la misma forma otros investigadores como Russell A. Berkeley (Cit. por 8) se centran en fallas en el control de estímulos presente en los niños con TDAH, lo que se relaciona con una obstaculización del procedimiento normal de aprendizaje, refiriéndose a una gran dificultad del niño para autorregular las respuestas ante estímulos del ambiente, incapacidad para generar un comportamiento que se mantenga en el tiempo y que no requiera de inspección externa; en este sentido Berkeley le asigna gran importancia a las funciones ejecutivas en la inhibición de conductas y autorregulación (implicaciones del lóbulo frontal) dado que entre estas se encuentra la memoria de trabajo, la motivación y la reorganización interna, siendo esta ultima la capacidad del lenguaje para representar objetos, acciones y propiedades que existen en el medio que conllevan a la internalización de conocimientos e información (8).

Es importante resaltar que el lenguaje es la herramienta fundamental del aprendizaje académico, en especial de la lectoescritura y la comprensión lectora. De esta manera y como afirman Azcoaga J, Eslava J, Peña E, Quintanar L. (15) un niño que presente alteraciones en su desarrollo del lenguaje llegara con esa herramienta defectuosa al aprendizaje pedagógico, poniéndose en gran riesgo de sufrir trastornos del aprendizaje cuando esas dificultades se tornen disarmónicas con las exigencias escolares. Por esta razón, la evaluación del lenguaje y de la competencia lingüística son elementos centrales durante la evaluación de los trastornos de aprendizaje.

Muchas son las investigaciones que se están realizando en la actualidad en búsqueda de una explicación que involucre todos los aspectos estudiados en sus particularidades como los expuestos anteriormente, el camino por recorrer es largo aun para encontrar soluciones y métodos para diagnosticar e intervenir niños con TDAH de manera integral, abarcando las dificultades de aprendizaje y todo lo que ello implica, además de factores sociales, involucrando inevitablemente un trabajo interdisciplinar.

\section{METODOLOGÍA}

Para elaborar el presente estudio se retomaron los lineamientos metodológicos de la bibliometria. Se realizó una búsqueda de artículos investigativos relacionados con el Trastorno de Hiperactividad, también denominado Trastorno por Déficit Atencional con Hiperactividad (TDAH), así como sus correlaciones con las diversas dificultades de aprendizaje.

Para la elección de la base de datos, se tuvieron en cuenta varios filtros de selección; el filtro principal consistía en el tema base de los diversos artículos: TDAH, el cual debía presentar correlaciones directas con las dificultades de aprendizaje. Por otro lado los artículos debían ser de carácter investigativo que reflejara estudios relacionados con este trastorno, cuyos resultados serán relacionados posteriormente con bases teóricas.

Otro de los filtros establecidos fue la edad de los participantes de las investigaciones de los artículos escogidos; para lo cual se escogió un rango de edad que contemplaba la vida escolar, es decir aproximadamente desde los 4 hasta los 17 años de edad, esto con el fin de establecer cuáles son las principales dificultades a nivel escolar en esta etapa de la vida. Por último, los artículos debían ser publicados a partir del 2000 hasta 2011, con el fin de obtener información actualizada y a partir de ella generar respuestas ante muchas cuestiones que abarcan el trastorno de Hiperactividad. Como ultimo filtro, el país de origen de los artículos debía ser de habla hispana. Finalmente se realizó a partir de los artículos seleccionados, una base de datos que resumía la información más relevante de cada uno de ellos (Tablas 1), lo cual facilito posteriormente un análisis estadístico, favoreciendo de manera directa la interpretación de los resultados obtenidos y los procesos de discusión por medio del análisis de los indicadores de conclusión y su correlación con los demás filtros mencionados anteriormente.

\begin{tabular}{|c|c|}
\hline 1 & $\begin{array}{l}\text { Comorbilidad entre el TDAH y trastornos del lenguaje en sus } \\
\text { diferentes componentes (forma, contenido y uso) y modalidades } \\
\text { (oral y escrito). }\end{array}$ \\
\hline 2 & $\begin{array}{l}\text { Comorbilidad entre el TDAH y dificultades de aprendizaje y/o bajo } \\
\text { rendimiento escolar. }\end{array}$ \\
\hline 3 & $\begin{array}{l}\text { Comorbilidad entre el TDAH y dificultades en la memoria (de } \\
\text { trabajo, a largo plazo, verbal y auditiva). }\end{array}$ \\
\hline 4 & $\begin{array}{l}\text { Presencia de alteraciones en la atención (alteraciones en el filtro } \\
\text { atencional, atención dividida y sostenida). }\end{array}$ \\
\hline 5 & $\begin{array}{l}\text { Diferencias significativas en los niveles de adaptación social } \\
\text { (pocas relaciones inter e intrapersonales) y escolar. }\end{array}$ \\
\hline 6 & $\begin{array}{l}\text { Alteración en las habilidades ejecutivas, la metacognición, la } \\
\text { conducta y la autorregulación. }\end{array}$ \\
\hline 7 & $\begin{array}{l}\text { Es necesario desarrollar una intervención interdisciplinaria para } \\
\text { mejorar las necesidades del individuo y fortalecer sus habilidades } \\
\text { con el fin de mejorar la calidad de vida. }\end{array}$ \\
\hline 8 & $\begin{array}{l}\text { Cambio positivo en la atención verbal y auditiva, tras la } \\
\text { intervención. }\end{array}$ \\
\hline 9 & $\begin{array}{l}\text { Presencia de efectos positivos y negativos en el aspecto cognitivo, } \\
\text { como efecto secundario en los tratamientos farmacológicos. }\end{array}$ \\
\hline 10 & $\begin{array}{l}\text { La maduración de los niños afecta positivamente la atención, la } \\
\text { memoria y la disminución de la hiperactividad. }\end{array}$ \\
\hline 11 & $\begin{array}{l}\text { Existen diferencias significativas en el desarrollo del TDAH entre } \\
\text { hombres y mujeres. }\end{array}$ \\
\hline 12 & $\begin{array}{l}\text { Existe relación entre los antecedentes personales, familiares y el } \\
\text { desarrollo del TDAH. }\end{array}$ \\
\hline
\end{tabular}

Tabla 1. Indicadores de conclusiones.

\section{RESULTADOS}

Se evidenció que las acciones profesionales más destacadas dentro de los 44 artículos seleccionados fueron la Evaluación (51\%) y la Intervención (27\%); mientras que las menos relevantes, en orden descendente, fueron el Diagnostico (14\%), seguido de la Consejería (4\%), y la Asesoría (4\%). Ninguno de los artículos se relaciona con la Promoción y la Prevención (figura 1.) 


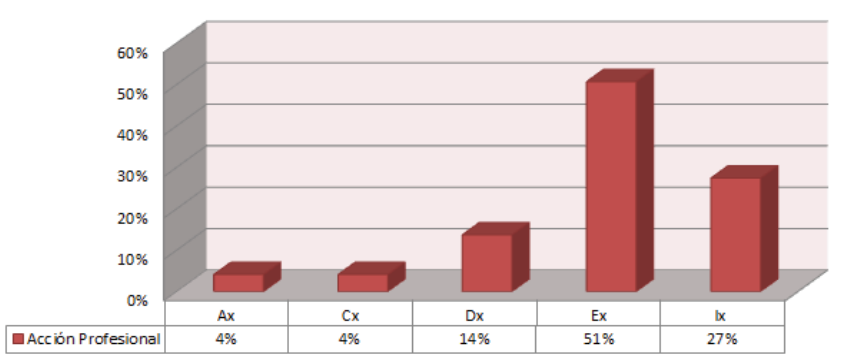

Figura 1. Prevalencia acción profesional.

Desde la perspectiva del año de publicación y presentando el mayor número de investigaciones con respecto al TDAH y su relación con los trastornos de aprendizaje, se destaca el año 2009 con el 20\%, seguido del 2008 con el $18 \%$, mientras que en 2000,2002 y 2003 se evidencio poco desarrollo investigativo (2\%) en el campo de la Hiperactividad, mientras que en los años restantes se encontró una tasa de variabilidad en investigación registrando (Figura 2).

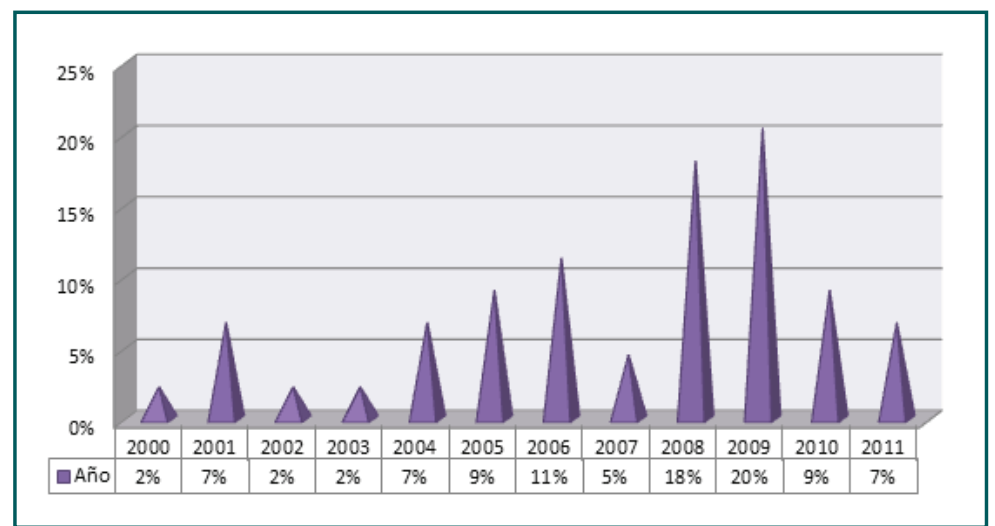

Figura 2. Prevalencia año.

En cuanto al enfoque teórico se evidencia que el Neuropsicológico es el predominante entre los autores debido a que el $40 \%$ de los artículos sustentan sus teorías basándose en los aportes de la Neuropsicología al campo del TDAH; seguido por el enfoque Cognitivo con el $26 \%$, el Psicosocial con $17 \%$, Conductual y Social con $6 \%$ cada uno, mientras que los menos empleados fueron el Lingüístico y Clínico representados únicamente por el 2\% (Figura 3).

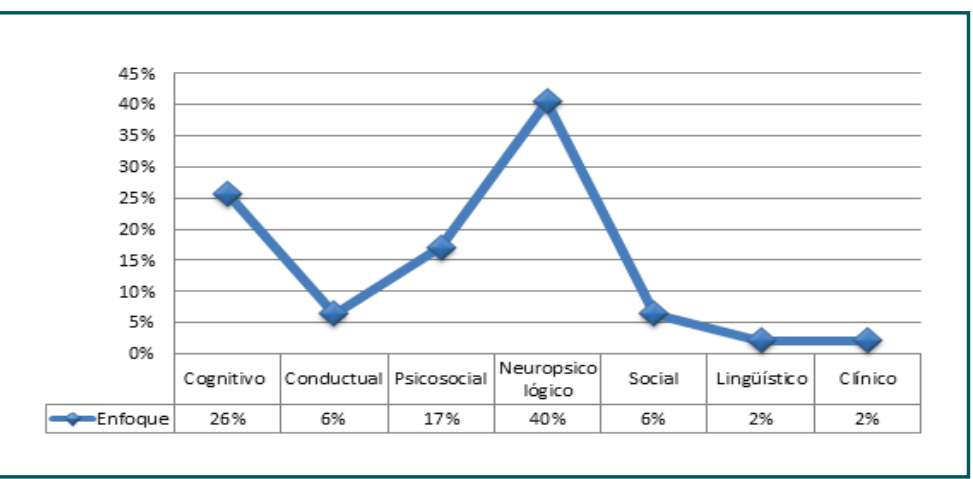

Figura 3. Prevalencia enfoque.
Con respecto a las conclusiones (Ver Tabla 1), los indicadores más relevantes fueron el 1, 2, 4 (16\%) los cuales se relacionan con la comorbilidad entre el TDAH y los Trastornos de lenguaje, las dificultades de aprendizaje y alteraciones en la atención; mientras que los indicadores restantes no fueron de gran interés para las investigaciones, relacionados estos con los cambios evidenciados tras la intervención, el tratamiento farmacológico y la maduración cerebral de los niños; como factores que influyen de manera positiva o negativa en la evolución del TDAH (Figura 4).

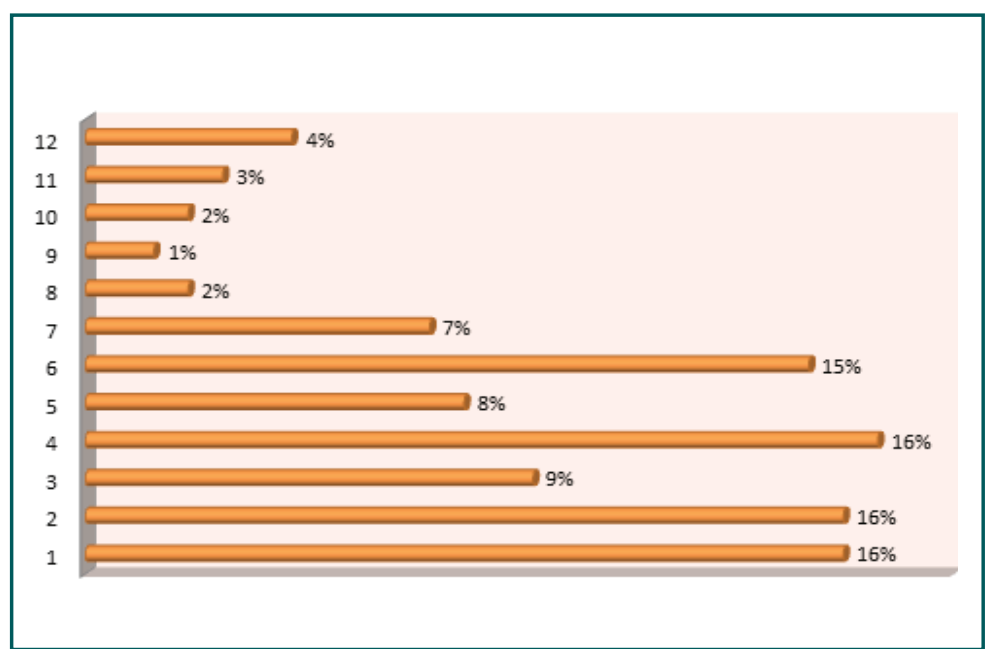

Figura 4. Prevalencia conclusiones.

A nivel del país se evidenció que el $46 \%$ de los artículos consultados provienen de España, es importante además resaltar el gran número de artículos provientes de Colombia, estipulados en el $23 \%$, seguidos por Puerto Rico y Cuba con el 4\%, los demás países no contaron con una muestra representativa mayor de 7,7\% (Figura 5).

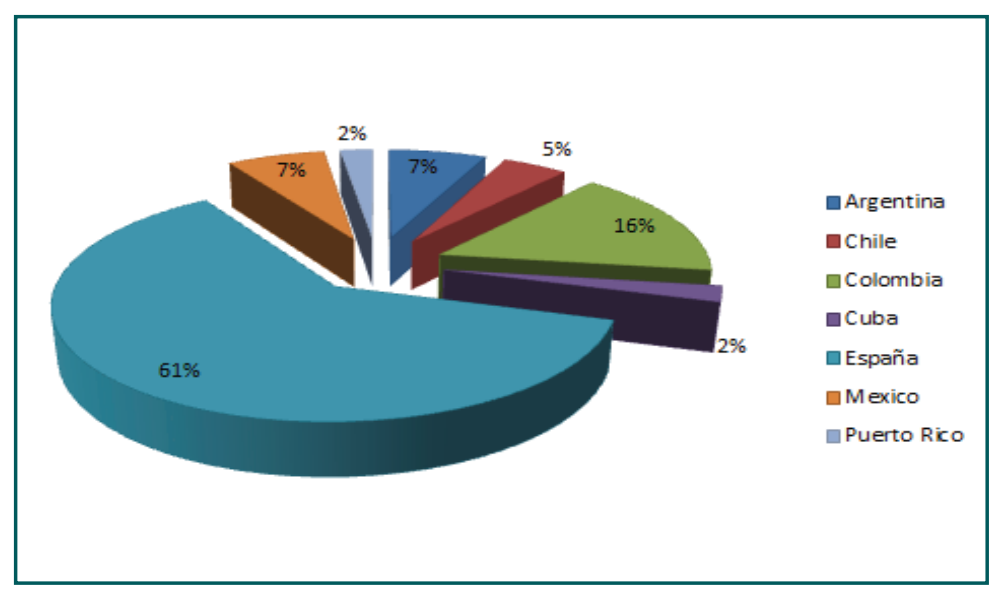

Figura 5. Prevalencia país.

En cuanto a la población se encontró que la mayoría de los artículos se centraba en niños que se encontraban entre los 7 y los 9 años de edad $(42.9 \%)$, seguido de los de 10 a los 12 (38.1\%), mientras que la población entre los 16 y 18 años fue la que menos se encontró en estos artículos con $2.4 \%$ (Figura 6). 


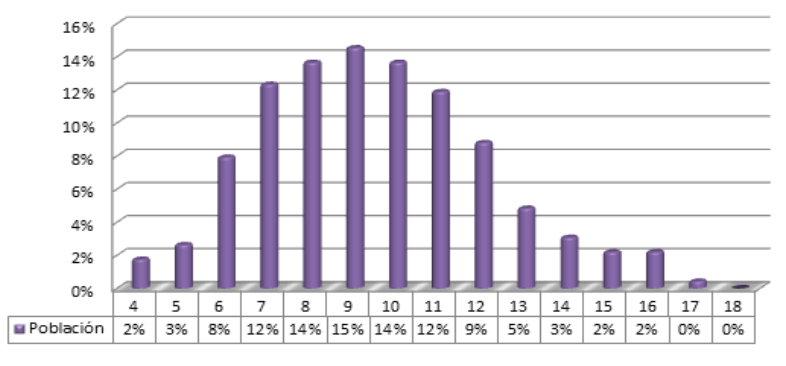

Figura 6. Prevalencia población.

\section{DISCUSIÓN}

El siguiente análisis se deriva del estudio minucioso de datos estadísticos que reflejan en términos porcentuales los resultados obtenidos, no solo de cada uno de los parámetros contemplados en este artículo (acción profesional, año, conclusiones, enfoque teórico, país y población) sino también de sus diversas confluencias.

Durante el 2008 y 2009 se reflejó un alto interés por la mayoría de las acciones profesionales, siendo las más relevantes la evaluación y la intervención, y en menor proporción consejería y asesoría, involucrando un incremento significativo en el conocimiento que se poseía con respecto a los diversos métodos de diagnostico y tratamiento ofreciendo a los profesionales interesados en el TDAH diversas alternativas para identificar y superar las múltiples dificultades características de este trastorno (las cuales fueron mencionadas anteriormente) siendo de vital importancia el interés incipiente de los investigadores por desarrollar intervenciones alternativas enfocándose en la reducción de los tratamientos farmacológicos que son implementados en el TDAH. Contrario a lo encontrado en periodos anteriores (2000 al 2007) donde las acciones profesionales no tenían mayor relevancia; implicando que durante este periodo de tiempo los aportes realizados desde la investigación al TDAH fueron incipientes en cuanto a la contribución a la detección y manejo de este trastorno.

Se encuentra además una preferencia hacia los enfoques neuropsicológico y cognitivo en el desarrollo de todas las acciones profesionales, especialmente en la evaluación; mientras que los otros enfoques tienen poca trascendencia, afectando de manera directa la percepción que se tiene del TDAH, debido a que al dejar de lado los enfoques social y el lingüístico se desmerita casi por completo el vital papel que desempeña el lenguaje y las relaciones sociales, las cuales como enunciamos anteriormente se encuentran afectadas en este trastorno; además al no ver al niño como un ser integral susceptible a sufrir alteraciones en diferentes niveles de la vida además del neuropsicológico, como son escolar y social, no se propende la calidad de vida de éste al entenderlo como un ser biomédico centrándose en disminuir los síntomas del trastorno; sin tener en cuenta el aspecto biopsicosocial del niño y su familia, dejando de lado sus intereses, expectativas y objetivos frente a los diversos aspectos involucrados en el TDAH. (16)

Los enfoques de mayor consideración, neuropsicológico y cognitivo, tienen una amplia relación con los indicadores 1 al 8 (ver
Tabla 1) los cuales hacen referencia a la comorbilidad del TDAH con los trastornos del lenguaje, las dificultades de aprendizaje, los problemas de atención y las diversas habilidades cognitivas, ejecutivas y lingüísticas alteradas en este trastorno; y son más comunes en estudios realizados con sujetos entre los 6 y los 13 años de edad; con respecto a este hecho se infiere que la aparición de los factores mencionados es más evidente en este rango de edad debido a que en esta se desarrolla gran parte de la etapa escolar facilitando su detección por parte de los familiares y los docentes involucrados en la educación del niño.

Así mismo se observa que el enfoque neuropsicológico es el preferido en la muestra de países, a excepción de México, que además es el único país donde hay presencia del enfoque psicosocial. Se advierte además que el enfoque lingüístico solo es manejado en España, con respecto a lo cual se infirió que esto puede estar relacionado con el hecho de que en este país se realiza un gran énfasis en el estudio de la lengua española siendo uno de sus grandes fuertes investigativos la lingüística. (17)

Continuando con los aportes provenientes de España, en los artículos realizados en este país se hace evidente un gran esfuerzo por describir de una manera muy detallada las falencias atencionales y cognitivas presentes en el TDAH además de relacionarlo directamente con las dificultades de aprendizaje, y en consecuencia, con el bajo rendimiento académico de estos niños. Situación similar es observada en Colombia, en donde el principal énfasis de investigación se observa en la descripción detallada de todas las dimensiones involucradas en el lenguaje, relacionando los diversos hallazgos realizados con el contexto escolar y social de los niños; lo cual, tiene poca relevancia en la mayoría de los países; este hecho hace que las investigaciones realizadas en el ámbito del TDAH en Colombia se destaquen a nivel internacional.

En cuanto a la caracterización de la población de niños con TDAH según su edad, se observó que en la población con un rango de edad entre los 4 a 7 años (a pesar de ser poco estudiada) es muy común encontrar problemas de lenguaje, deficiencias atencionales reflejadas en una inadecuada capacidad de sostener la atención y la presencia de distractibilidad; es importante destacar que los niños en esta etapa se encuentran en una transición al ámbito escolar lo cual implica un gran incremento en las demandas atencionales y cognitivas a las que se debe enfrentar; además no ha terminado de desarrollar de una manera integral el lenguaje, estos factores al encontrarse acompañados por el TDAH dificultan el aprendizaje y la adquisición del lenguaje. (18). Los niños con edades entre los 7 y los 13 años de edad, evidencian además de las características mencionadas anteriormente, dificultades de aprendizaje en general, manifestadas específicamente en discalculia, disgrafía, dislexia y un bajo rendimiento académico en general; lo cual es fácilmente detectado en esta etapa debido a que se inicia el procesos escolar de adquisición formal de los procesos lectoescritos, haciendo evidentes las dificultades en la adquisición de los mismos.

En cuanto a los indicadores de conclusión estudiados y su relación temporal, se evidencia que inicialmente en el año 2000 las investigaciones se inclinaban más a estipular qué dimensión del lenguaje se encontraba más afectada (concluyendo que la mayor deficiencia 
se encontraba en la forma y el contenido) y enfocándose en los problemas atencionales especialmente en la función deficiente del filtro atencional y la atención sostenida, siendo de poca relevancia su correlación con las dificultades de aprendizaje. Esta situación empieza a cambiar significativamente en el 2005, debido a que empieza a predominar la inclusión de la presencia de dificultades tanto en el lenguaje como en el aprendizaje y empiezan a describir de una manera más detallada las conductas asociadas al TDAH como lo son la falta de autorregulación y la inestabilidad conductual; lo cual interfiere en la eficiencia del proceso de selección y almacenamiento de la información relevante suministrada en el aula de clase, tendencia que es sostenida a través de los demás periodos temporales estudiados.

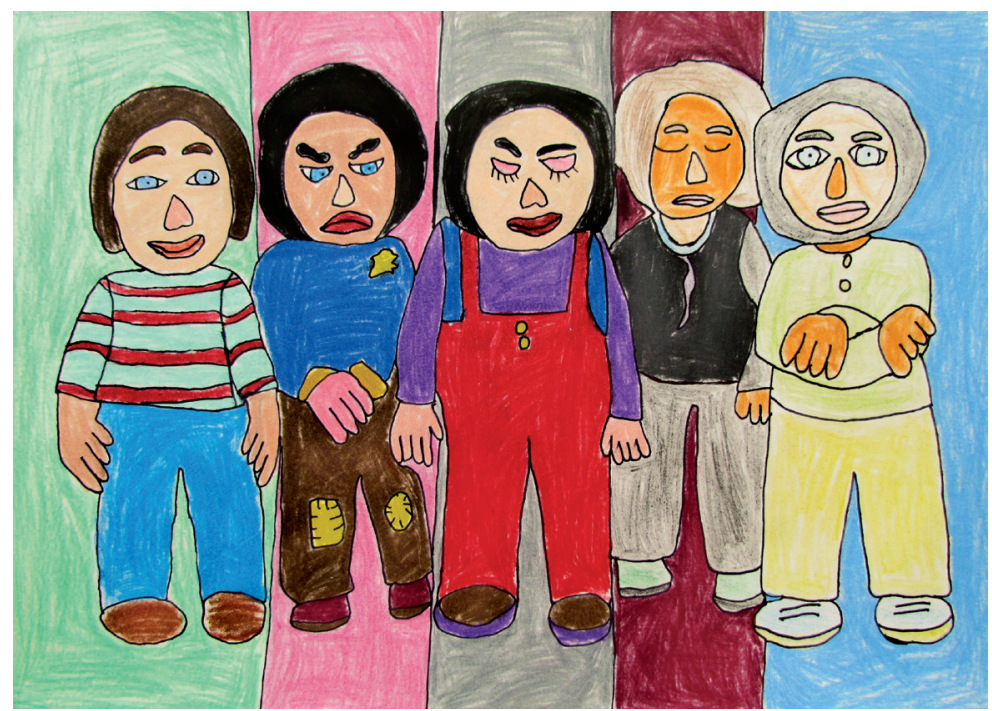

Andrés Ramírez - Arte sin Fronteras - Programa Talentos Especiales

Se ha observado que las dificultades de aprendizaje escolar muchas veces se fundamentan en un problema de base atencional, debido a que los niños desatentos (como es el caso de los niños con TDAH) no pueden realizar tareas fundamentales para el proceso de aprendizaje como por ejemplo seleccionar los estímulos relevantes de su ambiente (filtro atencional) y sostener la atención en un estímulo determinado durante un periodo de tiempo prolongado, a pesar de poseer un potencial intelectual normal; motivo por el cual, la información base captada por estos niños es deficiente desde un primer instante y la dificultad de aprendizaje se consolida en el momento de almacenar esta información deficiente en la memoria a largo plazo (MLP), debido a que además de existir una dificultad para "retener" la información, también se dificulta el proceso de recuperación y acceso a la misma (19). En el caso específico de los niños con Hiperactividad, como se ha mencionado anteriormente, se encuentran conductas como la impulsividad y obviamente la falta de atención, condiciones que los hace susceptibles a tener dificultades en diversas áreas como la cognitiva y social, lo cual interfiere directamente en el aprendizaje.

Con base en la presente revisión teórica e investigativa, se estableció una relación directa entre TDAH y su incidencia en las dificultades de aprendizaje, la cual radica principalmente en un déficit atencional y una serie de deficiencias cognitivas y metacognitivas que interfieren de manera directa en los procesos normales de re- cepción, selección, análisis, síntesis y almacenamiento de la información recibida, procesos que son de vital importancia en lo que se refiere al aprendizaje escolar.

El predominio de las investigaciones, realizadas con respecto al TDAH y su comorbilidad con trastornos de aprendizaje, a partir del año 2009, se relaciona con un aumento significativo en el interés de estudiar y analizar esta relación, la cual empieza a ser cada vez más relevante e incidente (9).

La prevalencia evidenciada en la mayoría de las investigaciones, hacia el uso de poblaciones comprendidas entre los 7 y los 11 años de edad puede correlacionarse con el hecho de que en estas edades el impacto de las dificultades tanto cognitivas como de aprendizaje se hacen más evidentes en diferentes ambientes, lo que permite además hacer detecciones tempranas y adecuadas, no solo de la presencia del trastorno, sino también de factores de riesgo, información clave para la elaboración de campañas de promoción y prevención.

La relevancia del análisis del TDAH desde perspectivas neuropsicológicas y cognitivas, es de vital importancia, debido a que a partir de estas se pueden derivar hipótesis que reflejan la incidencia de las dificultades de aprendizaje en los niños con este trastorno basándose en alteraciones a nivel biológico y cognitivo, estas últimas derivadas de fallas propias en la atención; lo cual complementa de una manera muy interesante tanto las hipótesis planteadas con respecto a las posibles causas como las conductas observadas en los niños con TDAH.

En cuanto a la participación de la Fonoaudiología, esta profesión tiene un papel muy importante en todos los procesos que involucren a la población con TDAH. Por un lado son cruciales en los procesos de evaluación ya que permiten un diagnostico adecuado y temprano, disminuyendo así el riesgo de dar diagnósticos apresurados e inapropiados. Las acciones de los Fonoaudiólogos son también importantes en el proceso de intervención, puesto que se ve al niño como un ser totalmente integral y bajo esta visión enfoca su tratamiento, cuyos resultados impactan positivamente en varios aspectos de la calidad de vida del niño y mejorara las competencias comunicativas.

También es necesario partir del hecho de que los niños con TDAH presentan en mayor y menor proporción, dificultades relacionadas con algún componente del lenguaje; dichas dificultades, no están dadas puramente por problemas lingüísticos en la mayoría de los casos, sino mas bien se originan en la suma de los efectos causados por el trastorno de hiperactividad, como lo son las alteraciones comportamentales, en las habilidades cognitivas y funciones ejecutivas; los cuales como se ha mencionado a lo largo del presente artículo inciden de manera directa en el desarrollo del lenguaje y a si mismo este influye de manera desfavorable en el aprendizaje y adecuado desarrollo lingüístico; con base en esto se hace evidente la necesidad de incluir dentro del equipo interdisciplinario de intervención de los niños con TDAH a la Fonoaudiología, como profesión especializada en el diagnostico y tratamiento de los trastornos del lenguaje, teniendo como base la capacidad de potencializar y recuperar estas habilidades basándose en teorías de neuroplasticidad cerebral, transformación de ambientes escolares y sociales, entre otras. 
Dicha transformación seria más provechosa si se contemplaran e implementaran nociones de la teoría planteada por Feuerstein sobre la Modificabilidad Cognitiva Estructural, específicamente partiendo desde un criterio de mediación cognitiva: La regulación de la conducta como se ha mencionado anteriormente es de gran importancia pues puede ejercer el papel de mediador de la interacción entre el docente y el niño en un ámbito escolar, lo que contribuye a aprovechar al máximo al análisis eficiente de la información. (20)

Otro criterio de mediación, contemplado por esta teoría está íntimamente relacionado con la potencialización de habilidades metacognitivas. La metacognición es crucial en los procesos de aprendizaje ya que permite que el sujeto conozca sus habilidades, reflexione sobre las diferentes procedimientos para enfrentarse a diversas tareas, qué estrategias cognitivas utiliza y qué tan efectivas son estas. $\mathrm{Al}$ desarrollar la metacognición en niños con TDAH por medio de la participación (constante inicialmente) de un mediador experto, se fomenta, a partir de la elección adecuada de estrategias y procesos de reflexión, la autorregulación del propio aprendizaje y de la conducta, herramientas cruciales y activas del proceso de aprendizaje; al lograr que el niño sea capaz de utilizar estrategias metacognitivas de manera eficiente, se logra además una independencia por parte de este, debido a que adquiere la capacidad de seleccionar y aplicar diferentes métodos seleccionados de manera consiente para solucionar los problemas planteados tanto en el ámbito escolar como en la vida diaria, sin necesidad de tener constantemente una guía por parte de un mediador.

Complementando las ideas planteadas anteriormente y partiendo de una base teórica según la cual los estilos cognitivos son constructos teóricos que explican procesos cognitivos atencionales, es decir, lo que ocurre en la mente del sujeto cuando éste elabora una respuesta frente a los estímulos ambientales, los procesa y se enfrenta a una tarea dada seleccionando diferentes estrategias para realizar la misma; la selección de estos estilos se realiza de manera consistente debido a que su raíz radica en una base metacognitiva (21). Teniendo en cuenta este concepto y extendiéndolo hacia el caso particular de los niños con TDAH, se puede observar una tendencia notable hacia un estilo cognitivo caracterizado por la impulsividad y la rigidez cognitiva, lo cual se refleja en una deficiencia para responder adecuadamente a los diversos requerimientos presentes en las tareas a las cuales se debe enfrentar, especialmente en el ámbito académico debido a la alta demanda presente en cuanto a la aplicación de habilidades metacognitivas, y al estar éstas limitadas por el estilo cognitivo mencionado anteriormente, se hace presente en la mayoría de los casos un bajo rendimiento escolar, muchas veces se debe fundamentalmente a la inflexibilidad y la aplicación estática de una sola estrategia cognitiva para resolver las actividades académicas además de una falencia clave de los procesos de planeación, autorregulación y evaluación de la efectividad de las mismas al concluir la tarea; evidenciándose una diferencia notable con la manera en la cual se desenvuelve un niño con un estilo cognitivo caracterizado por la reflexibilidad quienes muestran un desempeño claramente superior (21).

Continuado con el proceso terapéutico de los niños con TDAH y centrándose en el rol del Fonoaudiólogo, este deberá buscar durante el proceso de intervención un aumento en el nivel de flexibilidad cognitiva y realizar un acercamiento hacia un estilo cognitivo orientado hacia a la autorregulación y evaluación consiente y constante durante el proceso de resolución de tareas tanto en el ámbito académico como en la vida cotidiana; logrando así una mejoría del rendimiento académico de estos niños, lo cual es usualmente la mayor preocupación de los padres y maestros involucrados en el procesos de educación de los mismos.

Estos procesos de autorregulación también pueden manejarse a través de otros sistemas de intervención aplicables al TDAH. El neurofeedback, por ejemplo, es una técnica actualmente muy utilizada para este fin. Consiste en un análisis automático de la actividad eléctrica del cerebro (22), que posibilita a las personas darse cuenta de cómo es el funcionamiento de éste. El entrenamiento bajo esta técnica, permite aprender a regular dicho funcionamiento y mantenerlo por un tiempo determinado; esto se logra a partir del control en la velocidad de ondas cerebrales, beta y theta, encargadas del control de la impulsividad y de estados de creatividad e inconsciencia respectivamente (23). El dominio y regulación de la actividad de estas ondas generará mejores periodos de atención, bajas conductas impulsivas e hiperactivas $y$, consecuentemente, un mejor desempeño personal, social y escolar.

A pesar de que Colombia no es el país con más investigaciones, los resultados de estas demuestran el fuerte de este país en términos de evaluación (12\%); estos porcentajes aunque son significativos, son realmente insuficientes teniendo en cuenta las diferentes variables que deben ser estudiadas en el TDAH. Una de esas variables, y tal vez la que últimamente ha tenido más auge, es el impacto del tratamiento farmacológico. En este tema, existen diversas y muy variadas posiciones, hay quienes apoyan la monoterapia e incluso la politerapia, pero se ha demostrado que muchas veces la medicación se da de forma indiscriminada, sin contemplar todos los factores, culturales y psicosociales inmersos en la vida del niño con TDAH, llegando incluso a ser estos la etiología de trastornos y déficits secundarios y no como tal la solución a la disfunción cerebral mínima.

El contexto, cultural y psicosocial, es tomado como otra gran variable, es importante considerar que muchos niños que se ven involucrados en ambientes que no favorecen su desarrollo físico, motriz, cognitivo, social y escolar; pueden presentar sintomatología similar a los niños con TDAH, y por este motivo suelen ser categorizados erróneamente como hiperactivos, muchos de estos diagnósticos además, se basan en juicios subjetivos, tanto de padres y maestros, que por un lado, no comprenden la magnitud de la naturaleza del trastorno y sus alteraciones relacionadas, y por otro, contribuyen a que el problema se exacerbe, y en este sentido es significativo resaltar el trabajo de intervención de un equipo interdisciplinar que involucra a padres de familia y determinados profesionales, dotados con los saberes necesarios para abordar todos los aspectos que rodean al paciente, dado que este independientemente de su patología base, debe verse como un ser integral sensible al ambiente, a la interacción social y a situaciones de diversa índole; y no solamente como sujeto experimental utilizado para conglomerar conocimientos basados en su situación particular.

El equipo en conjunto deberá tomar en cuenta que se debe buscar el bienestar social, escolar y familiar del niño, es importante que se 
aborden los diferentes problemas desde los diversos puntos de vista y desde diferentes modelos de intervención que se pueden complementar entre sí, contemplando el trastorno presentado como una alteración del sujeto, la cual está siempre mediada e influenciada por su cotidianidad y realidad social; así mismo es necesario integrar las habilidades y capacidades que demuestra el niño para relacionarlo con mayor facilidad al entorno de aprendizaje escolar. El apoyo a los niños, niñas y adolescentes con TDAH demanda la cobertura de servicios necesarios para garantizar el adecuado desarrollo social, emocional, y conductual; esto incluye, contemplar las necesidades: médica, farmacéutica, terapéutica y de educación, siendo estas prioritarias para el bienestar y mejoramiento de la calidad de vida; con este fin se desarrolló el proyecto de ley 216 del 2009 del Congreso de Colombia, en el cual se busca cubrir estas necesidades sin importar el nivel socio-económico, facilitando una mejor atención, apoyo integral y multidisciplinar, regido por el desarrollo personal, igualdad y dignidad humana, permitiendo la inclusión social y un mejor futuro para las personas con TDAH (para mayor información remitirse a http://www.gobiernobogota.gov.co/).

\section{CONCLUSIONES}

Por medio de la presente investigación se realizó un exhaustivo esfuerzo por analizar los diferentes puntos de encuentro entre el TDAH y las dificultades de aprendizaje, encontrando diversas posturas y enfoques teóricos, los cuales contribuyeron de manera directa a la conformación de un perfil integral descrito detalladamente en el transcurso de este estudio.

Con el fin de contemplar la influencia de los problemas comorbidos al TDAH desde un panorama más amplio incluso en el momento de referirse al trastorno en sí mismo; consideramos importante tener en cuenta las diversas dimensiones de la vida que se ven afectadas en estos niños, teniendo en cuenta esta idea llegamos a la conclusión de que es importante que las investigaciones se contemplen bajo los diversos enfoques teóricos desde los cuales se puede contemplar el TDAH, y no solo a partir del neuropsicológico o cognitivo, lo cual como es evidente en la presente investigación, es la tendencia actual de las investigaciones.

Partiendo de la noción de interdisciplinariedad, y refiriéndonos desde una visión centrada en la Fonoaudiología, se considera que teniendo en cuenta todos los factores nombrados y analizados durante el curso de esta investigación, esta profesión debería ser participe activa de todos los procesos clínicos, rehabilitativos, adaptativos e incluso investigativos, inherentes de los sujetos con TDAH; esto basándonos en los múltiples conocimientos en campos alterados en estos niños como las habilidades cognitivas, las dificultades de aprendizaje, las deficiencias en el procesamiento matemático, la lectura, la escritura y especialmente el lenguaje; siendo todos estos temas abordados ampliamente y con profundidad desde la Fonoaudiología, justificando así la valiosa e importante participación de estos profesionales en los procesos clínicos y de intervención relacionados con el TDAH y trastornos asociados.

Aunque las investigaciones actuales, como fue evidente a través de la presente investigación, con el paso del tiempo son más significativas, no solo en términos de cantidad sino también de resultados y aplicabilidad, se considera que es necesario que se realicen más estudios que involucren diferentes contextos y que contemplen la interdisciplinariedad como un factor primordial en los procesos de evaluación, diagnostico, pero sobre todo de intervención. 


\section{Referencia:}

Las referencias a otras obras son una parte muy importante en la literatura científica;
ya que estas permiten conocer más sobre los autores y
mantener vivas sus voces dentro del texto.

1. Álvarez D, Álvarez L, Bernardo A, García, González J, González P, Núñez J, Rodríguez C. TDAH y dificultades de aprendizaje en escritura: comorbilidad en base a la atención y memoria operativa. European Journal of Education and Psychology, España: 2009 Vol. 2, pág. 181198.

2. Álvarez D, Álvarez L, Bernardo A, Gazquez J, García J, Gonzales J, Gonzales P, Núñez J, Rodríguez C. El proceso de revisión escrita en alumnos con trastorno de déficit de atención e hiperactividad y dificultades de aprendizaje. Revista Psicodidactica, España: 2009 Vol. 14, pág. 279-291.

3. Aguirre A, Gómez L, Pineda D. Conciencia fonológica en niños con trastorno de la atención sin dificultades de aprendizaje. Revista de Neurología, España: 2005 Vol. 40, pág. 581-586.

4. Bara S, Henao G, Pineda D, Vicuña P. Perfiles neuropsicológicos y conductuales de niños con trastornos por déficit de atención/hiperactividad de Cali, Colombia. Revista de Neurología, España: 2003 Vol. 37, pág. $608-615$

5. Warnock M. Special education needs. Informe de la Comisión de Investigación sobre la educación de niños y jóvenes discapacitados. Her Majesty's Stationery Office. Londres: 1978

6. Acle G, Olmos A. Problemas de Aprendizaje: Enfoques Teóricos. Facultad de Estudios Superiores Zaragoza, UNAM. 2005

7. Alonso M, Gonzalez P, Gonzalez R, Hernández S, Izquierdo M, Quintero l, Rubio B. Evaluación neuropsicológica de la memoria en el trastorno por déficit de atención/hiperactividad: papel de las funciones ejecutivas. Revista de Neurología, España: 2008 Vol. 47, pág 225-230.

8. Servera M. Modelo de autorregulación de Barkley aplicado al trastorno por déficit de atención con hiperactividad: una revisión. Revista de Neurologia, España: 2005. Vol.40, 358-368.

9. Guerrero D, Jiménez M, López M. Relación entre la experiencia óptima percibida y rendimiento académico según la presencia del TDAH. Revista Mexicana de Psicología. México: 2010. Vol. 27, pág. 15 - 23.

10. Arán V, Mias C. Neuropsicología del trastorno por déficit de atención/ hiperactividad: subtipos predominio déficit de atención y predominio hiperactivo compulsivo. Revista Argentina de Neuropsicología, Argentina, 2009 Vol. 13, pág. 14-28

11. Jarque S, Gil-Llarioa M, Miranda-Casasa A, Uribea L. Evaluación e intervención en niños preescolares con manifestaciones de trastorno por déficit de atención con hiperactividad y conducta disruptiva. Revista de Neurología, España: 2003 Vol 36, pág. 85 - 94.

12. Barceló E, Pineda D, Puentes P. Características conductuales y neuropsicológicas de niños de ambos sexos, de 6 a 11 años con trastorno por déficit de atención/hiperactividad. Revista de Neurología. 2008. Vol. 47, 175-184

13. Aguilar L, Rodríguez R. Trastornos de la atención y hallazgos electroencefalográficos en niños con crisis parciales complejas. Revista Habanera de Ciencias Medicas, Cuba: 2008. Vol. 7 .

14. Pascual, I. (2009).Síndrome de déficit atención-hiperactividad. Madrid: Ediciones Días de Santos.

15. Azcoaga J, Eslava J, Peña E, Quintanar L. Los trastornos de aprendizaje: perspectivas neuropsicológicas. Editorial Magisterio. Universidad Autónoma de Puebla.Méxivo. 2008

16. Borrell F. El modelo biopsicosocial en evolución. Revista de Medicina Clínica; España: 2010. Vol. 119 (5); pág. 175 - 179.

17. Galindo F. Enfoque lingüístico. Instituto Politécnico Nacional; México: 1994.Disponible en: www.fgalindosoria.com.

18. Casas A, De Alba A, Mulas F, Rosselló B, Taverner R. Dificultades en el aprendizaje de matemáticas e niños con trastorno por déficit de atención e hiperactividad. Revista de Neurologia, España<. 2009. Vol. 42, pág. $163-170$

19. Moreno M, Rodríguez W. El uso del lenguaje en niños y niñas con características del trastorno por déficit de atención con hiperactividad durante la planificación de una tarea. Revista Interamericana de Psicología, Puerto Rico:2011. Vol. 35, pág.142-162.

20. Velarde E. La Teoría de la Modifiacabilidad estructural cognitiva de Reuven Feuerstein. Investigación Educativa. 2008. Vol 12 (22). 203 211.

21. Gargallo B. Un Programa De Intervención Para Mejorar La Reflexividad En Preadolescentes ( $8^{\circ}$ De EGB Primaria); Revista Española de Pedagogía; España: 1987. Vol. 184; pág. 539-555.

22. Braidot, N. Neuromarketing: ¿Por qué tus clientes se acuestan con otro si dicen que les gustas tú? Argentina: 2009. pág. 102.

23. Alvarez L, Bernardo A, Gonzalez J, Gonzalez P, Nuñez C. Evaluación y control de la activación cortical en los déficit de atención sostenida. International Journal of Clinical and Health Psychology, España: 2007. Vol. 8, pág. 509-524. 